\title{
Inside Attack Filtering for Robust Sensor Localization
}

\author{
Jongho Won \\ Department of Computer Science \\ Purdue University, USA \\ won12@purdue.edu
}

\author{
Elisa Bertino \\ Department of Computer Science \\ Purdue University, USA \\ bertino@purdue.edu
}

\begin{abstract}
Several solutions have recently been proposed to securely estimate sensor positions even when there is malicious location information which distorts the estimate. Some of those solutions are based on the Minimum Mean Square Estimation (MMSE) methods which efficiently estimate sensor positions. Although such solutions can filter out most of malicious information, if an attacker knows the position of a target sensor, the attacker can significantly alter the position information. In this paper, we introduce such a new attack, called Inside-Attack, and a technique that is able to detect and filter out malicious location information. Based on this technique, we propose an algorithm to effectively estimate sensor positions. We illustrate the impact of inside attacks on the existing algorithms and report simulation results concerning our algorithm.
\end{abstract}

\section{Keywords}

Localization; Security; Wireless Sensor Networks

\section{INTRODUCTION}

Wireless Sensor Networks (WSNs) play a critical role in many domains by providing sensing and monitoring services. Their applications range from military tasks to civilian tasks such as surveillance, fire detection and soil condition monitoring for agriculture. In these applications, the location information of sensors is essential to identify the origins of events or sensed data. In addition, location information is critical for many system functions, such as geographic routing [6] and location-based key management [9]. However, in many cases, the location of sensors can only be determined after deployment since sensors may be deployed via random scattering (e.g., from an airplane). Sometimes, their initial locations can change due to natural phenomena such as wind or rain, or be moved by users or other agents (e.g., mobile sensors). Equipping each sensor with a Global Positioning System (GPS) receiver is undesirable due to the high cost. Therefore, several schemes for sensor localization

Permission to make digital or hard copies of all or part of this work for personal or classroom use is granted without fee provided that copies are not made or distributed for profit or commercial advantage and that copies bear this notice and the full citation on the first page. Copyrights for components of this work owned by others than ACM must be honored. Abstracting with credit is permitted. To copy otherwise, or republish, to post on servers or to redistribute to lists, requires prior specific permission and/or a fee. Request permissions from permissions@ acm.org.

ASIA CCS'16, May 30-June 3, 2016, Xi'an, China.

(C) 2016 ACM. ISBN 978-1-4503-4233-9/16/05 . \$ $\$ 15.00$

DOI: http://dx.doi.org/10.1145/2897845.2897926 have been proposed based on the use of a small number of beacon (or anchor) nodes which know their positions. Such schemes estimate sensor locations using various information received from beacon nodes such as beacon nodes' positions, distances from beacon nodes, angles of beacon signals or connectivity information.

However, if sensors are deployed in an unattended hostile area, the estimated locations can be severely distorted by even simple attacks such as replay attacks. For example, if the distance between a sensor and a beacon node is measured by the Received Signal Strength Indictor (RSSI), an attacker can reduce the distance by replaying beacon signals with a strong signal strength. As a result, the attacker can break the location-based functions of WSNs.

In the last decade, various localization schemes have been proposed for use in adversarial environments. However, some schemes are not well-suited for general WSNs since they require additional hardware such as directional antenna [7] or ultra-sound [3]. To tackle these problems, several secure localization schemes based on Minimum Mean Square Estimation (MMSE) have been proposed [8, 10, 12]. Since they utilize a simple MMSE method [11], their computational and storage costs are low enough to be successfully implemented on real sensor platforms. However, we found that an attacker can considerably degrade the estimation accuracy if the positions of victim sensors are known to the attacker.

In this paper, we first introduce such a new attack, called Inside-Attack, which severely degrades the estimation accuracy of most MMSE-based schemes. To defend against such attacks, we propose an Inside-Attack Filtering (IAF) algorithm which filters malicious location information introduced by the inside-attack. Then, we present a sensor localization algorithm, called IAF-MMSE, which significantly reduces the computational overhead and improves the estimation accuracy compared to other MMSE-based schemes.

\section{RELATED WORK}

Secure localization schemes based on MMSE utilize location information, called location reference, which consists of the position information $\left(x_{i}, y_{i}\right)$ of a beacon node $i$ and the distance $d_{i}$ between the sensor and the beacon node $i$.

Liu et al. [10] proposed three schemes based on MMSE. Such schemes filter out bad information and try to find out a subset of location references which produces the minimum mean square error by iteratively performing a MMSE. The three MMSE-based schemes have different search strategies. The first scheme, called Brute-Force MMSE (BF-MMSE), is based on a brute-force search. It iteratively executes the 
MMSE with all possible subsets of location references from the largest subset to the smallest subset until a result of the MMSE is lower than a threshold. Although BF-MMSE outputs relatively more accurate sensor positions than the other two MMSE-based schemes, it suffers from high computational overhead when the number of location references is large. The second scheme, called Greedy Attack Resistant MMSE (GAR-MMSE), is based on a greedy algorithm. Rather than performing the MMSE for all possible subsets, it drops one potentially malicious location reference for each round. Although this scheme is more efficient than the brute-forth scheme, it is weak against collusion attacks. The third MMSE-based scheme, called Enhanced-greedy Attack Resistant MMSE (EAR-MMSE), utilizes the fact that benign location references intersect each other. Each location reference has a counter which is the number of other location references intersecting with itself. Like the greedy algorithms, it drops a location reference which has the lowest count per round. EAR-MMSE is the most efficient in terms of computational and storage cost compared to any other schemes. In addition, the accuracy of EAR-MMSE is higher than the accuracy of the greedy algorithm and comparable to BF-MMSE. However, its localization accuracy dramatically decreases when an attacker knows the true positions of sensors as discussed later.

Wang et al. [12] developed the Cluster-Based MMSE (CMMSE) which tries to cluster benign location references by randomly selecting two location references as seeds. It reduces the time required to estimate a sensor position compared to EAR-MMSE by sacrificing the estimate accuracy.

A preliminary version of some of the work reported here was presented in a poster paper [14]. However, the poster paper only introduces an example of the Inside-Attack and rough ideas for a defense scheme.

\section{BACKGROUND}

In this section, we first describe network and threat models. We then provide a brief overview of the MMSE and the notion of Degree of Consistency.

\subsection{Network Model}

In our network model, there are two kinds of nodes: beacon nodes and non-beacon nodes. Beacon nodes know their locations because they can directly obtain their current location information from other sources such as GPS receivers or manual inputs. In contrast, the locations of non-beacon nodes are unknown and have to be estimated by using beacon nodes. In what follow we use the term sensor as synonym for non-beacon node.

A beacon node sends a beacon signal which includes the beacon node's identity, the current time, and its current location information. After a sensor receives several beacon signals from different beacon nodes, it measures the distances from the beacon nodes using a distance measurement method such as the Received Signal Strength Indicator (RSSI) or the Time Difference on Arrival. A sensor collects a set of location references for its position estimation. A location reference consists of a triple $\left\{x_{i}, y_{i}, d_{i}\right\}$ where $\left(x_{i}, y_{i}\right)$ is the position of the beacon node $i$ and $d_{i}$ is the measured distance. Since sensors are usually low-end embedded devices that are battery-powered, the computational cost for localization should be minimized. We assume that distance measurement errors are uniformly distributed in $[-\epsilon, \epsilon]$.

\subsection{Threat Model}

The goal of the attacker is to make the estimated locations of sensors far away from their true locations. We assume that the attacker is able to manipulate any element of a location reference $\left\{x_{i}, y_{i}, d_{i}\right\}$ by performing various attacks such as beacon node compromises, worm hole attacks and replaying beacon signals for distance reduction/enlargement. For example, if a beacon node is compromised, it can manipulate any element of a location reference. Since the beacon node has the secret key, the malicious location reference is successfully authenticated by sensors. In case of worm hole attacks and replay attacks, the attacker can just modify $d_{i}$. For instance, the attacker can reduce $d_{i}$ by replaying beacon signals with increased signal strength.

\subsection{Minimum Mean Square Estimation}

Suppose that a sensor obtains a set $\left\{l_{1}, l_{2}, \ldots, l_{n}\right\}$ of $n$ location references from beacon nodes, where $l_{i}=\left(x_{i}, y_{i}, d_{i}\right)$, and the estimated location is $(\hat{x}, \hat{y})$. Then, the mean square error $\delta$ of this location estimation is as follows:

$$
\delta=\frac{1}{n} \sum_{i=1}^{n}\left(d_{i}-\sqrt{\left(\hat{x}-x_{i}\right)^{2}+\left(\hat{y}-y_{i}\right)^{2}}\right)^{2}
$$

MMSE is a method which obtains the estimate $(\hat{x}, \hat{y})$ by minimizing $\delta$. MMSE is a nonlinear least squares problem which is solved by well-known optimization methods like the Gauss-Newton method [4], the Gradient descent method [5] and the basic MMSE [11]. These methods also involves complex calculations such as Jacobian matrix formation or matrix multiplications, and their computational complexity increases as $n$ increases. Therefore, when a sensor localization algorithm is designed, the number of MMSE operations should be minimized.

\subsection{Degree Of Consistency}

Our algorithm utilizes the notion of Degree Of Consistency to filter out malicious location references.

Definition 1. (Degree Of Consistency [10]) Each location reference $l_{i}$ has a counter called Degree Of Consistency (DOC). The DOC of $l_{i}$ is the number of other location references $\left(l_{j}, i \neq j\right)$ which intersect $l_{i}$.

The way to check whether two location references $l_{i}$ and $l_{j}$ intersect is as follows. Considering the maximum measurement error $\epsilon$, there are two circles for a location reference. For instance, the two circles of $l_{i}$ are $\left\{x_{i}, y_{i}, d_{i}-\epsilon\right\}$ and $\left\{x_{i}, y_{i}, d_{i}+\epsilon\right\}$. Let $d_{i j}$ be the distance between two beacon points, i.e., $\sqrt{\left(x_{i}-x_{j}\right)^{2}+\left(y_{i}-y_{j}\right)^{2}}$. If the following three conditions are all true, $l_{i}$ and $l_{j}$ do not intersect: (1) $d_{i j}>\left(d_{i}+\epsilon\right)+\left(d_{j}+\epsilon\right),(2) d_{i j}+\left(d_{i}+\epsilon\right)<\left(d_{j}-\epsilon\right)$ and $(3)$ $d_{i j}+\left(d_{j}+\epsilon\right)<\left(d_{i}-\epsilon\right)$. Otherwise, they intersect.

\section{INSIDE ATTACK}

In this section, we introduce a new attack called Inside Attack which causes serious estimation errors in EAR-MMSE.

\subsection{Inside Attack}

The DOC is utilized to filter out malicious location references and this filtering makes MMSE-based localization algorithms efficient [10]. As shown in Fig. 1, the number of DOCs of benign location references $\left(l_{1}, l_{2}\right.$ and $\left.l_{3}\right)$ are greater than or equal to 2 , whereas the malicious location references 


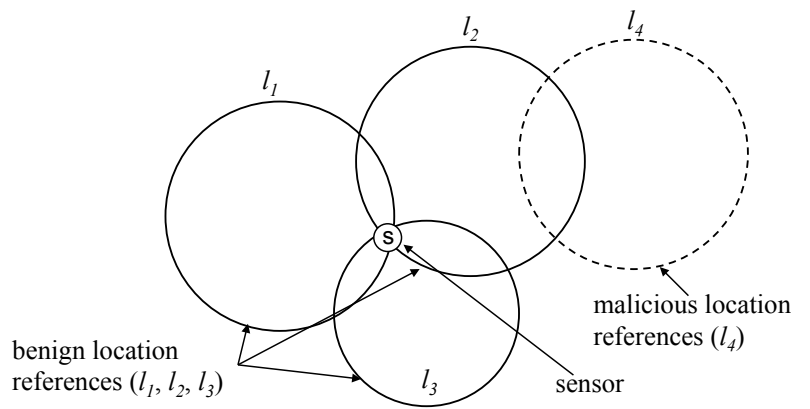

Figure 1: Outside attack example. The DOCs of $l_{1}$ and $l_{3}$ are equal to 2. The DOC of $l_{2}$ is 3 . The DOC of $l_{4}$ is 1 .

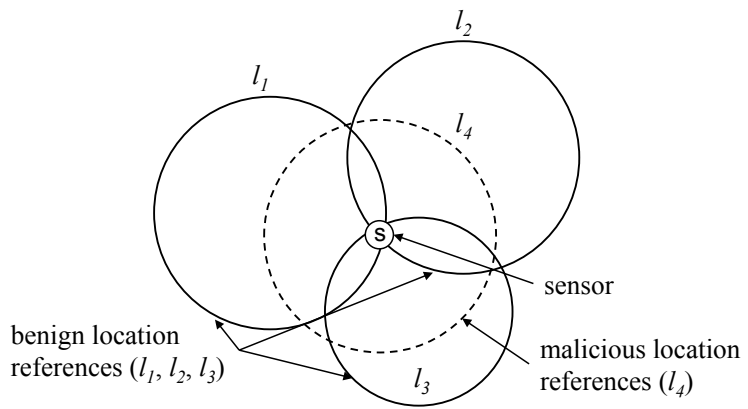

Figure 2: Inside attack example 1. The DOCs of $l_{1}, l_{2}, l_{3}$ and $l_{4}$ are equal to 3 .

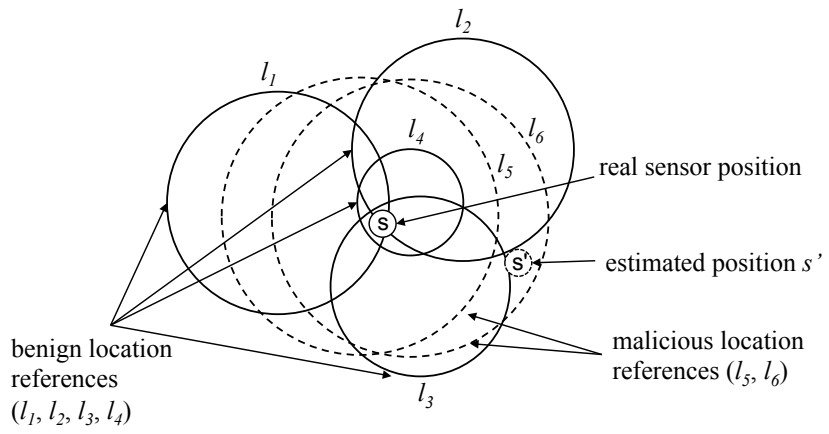

Figure 3: Inside attack example 2. The DOCs of $l_{1}, l_{2}$ and $l_{3}$ are equal to 5. The DOCs of $l_{5}$ and $l_{6}$ are equal to 4. The DOC of $l_{4}$ is equal to 3 .

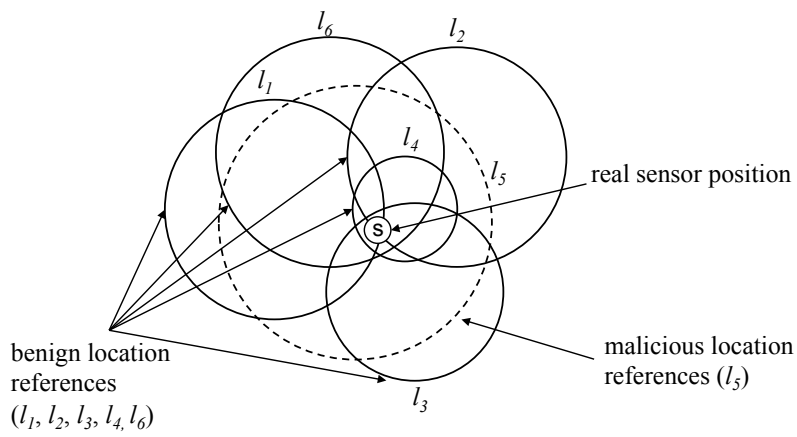

Figure 4: Inside attack example 3.

$l_{4}$ has a DOC of 1 . Therefore, if a malicious location reference is far from the true location of a target sensor, it will be removed. Now assume that a malicious location reference includes the true position of a sensor as shown in
Fig. 2. In this case, all location references $\left(l_{1}, l_{2}, l_{3}\right.$ and $\left.l_{4}\right)$ have a DOC of 3. However, EAR-MMSE [10] does not have a specific tie-break rule and must randomly choose one of them.

If there are two malicious location references, EAR-MMSE may remove a benign location reference as shown in Fig. 3. Since the DOC of the benign location reference $l_{4}$ is the lowest DOC, i.e., $3, l_{4}$ is removed in the first iteration. Then the DOCs of all location references become equal to 4 and one of remaining five location references will be removed. If a benign location reference, e.g., $l_{1}$, is removed, the estimated position will be $s^{\prime}$ with a high probability. We call such a malicious location reference inside-attack reference which includes the true sensor position and does not have the lowest DOC. The inside attack is an attack causing an estimation error using inside-attack references.

In order for an inside attack to be successful, an attacker has to generate an inside-attack reference which satisfies the following two conditions.

1. $m$ inside-attack references must intersect at least $n-2 m$ benign references ( $n$ is the total number of references).

2. The $m$ inside-attack references must intersect each other.

If the two conditions are satisfied, the DOCs of the insideattack references and the victim benign references are all equal to $n-m-1$. For example, assume there are four benign references and three inside-attack references $(n=7$ and $m=3$ ). Since four benign references intersect each other, their DOCs are at least 3. Also, since the three inside-attack references intersect each other, their DOCs are at least 2. Now, if the three inside-attack references intersect with $1(=n-2 m=7-2 \times 3)$ benign reference, e.g., $l_{1}$, then, the DOCs of the inside-attack references becomes 3 and the DOCs of $l_{1}$ becomes 6 . Since the DOCs of benign references, except $l_{1}$, are also 3 , one of six references (three inside-attack references and three benign references) will be randomly removed. If a benign reference is removed, the DOCs of all remaining benign references will decrease by 1 , while the DOCs of three inside-attack references will not change. Therefore, in the next iteration, one of benign references, except $l_{1}$, will be removed. Finally, one benign reference and three inside-attack references will remain. This procedure is illustrated as follows: $\{6,3,3,3,3,3,3\} \rightarrow\{5, *, 2,2,3,3,3\} \rightarrow\{4, *, *, 1,3,3,3\} \rightarrow$ $\{3, *, *, *, 3,3,3\} \rightarrow \cdots$, where $\left\{D O C_{1}, D O C_{2}, \ldots, D O C_{7}\right\}$ are the DOCs of the references and $D O C_{5}, D O C_{6}$ and $D O C_{7}$ are the DOCs of inside-attack references. * means that the reference is removed.

However, not all inside-attack references are harmful. If the circle line of an inside-attack reference is close to the true sensor position $\left(x_{0}, y_{0}\right)$, it is not different from a benign reference. Therefore, to make an inside-attack reference harmful, the circle lines of the inside-attack references should be far from the true sensor position. In other words, an inside-attack reference with $\left(x_{0}, y_{0}, d_{\max }\right)$ is most malicious if it satisfies two conditions above. Here, $d_{\max }$ is the maximum measurement distance.

An inside attacker can easily find the true position of a victim sensor by deploying hidden nodes around a target sensor $t$. The hidden hodes can measure the distances from $t$ by overhearing messages sent by $t$. The attacker then estimates the sensor position by solving MMSE. Possible estimation 
errors which an inside-attacker can cause are bound to $d_{\max }$. However, if the time-of-flights of RF signals (e.g, GPS) or the RSSI using powerful antennas $((>1 \mathrm{~km})[1])$ is utilized as a distant measurement method in order to send/receive beacon signals from afar, the attacker's capability is not negligible since $d_{\max }$ becomes very large.

\section{DEFENSE SCHEME}

In this section, we first propose an Inside-Attack Filtering (IAF) algorithm which filters out inside-attack references. Then, we present a secure localization algorithm, called IAFMMSE, which efficiently estimates sensor positions using $\mathrm{IAF}$ and the DOC.

\subsection{Inside Attack Filtering Algorithm}

Our IAF algorithm utilizes the two following facts:

- A harmful inside-attack reference $l_{i}$ has a large $d_{i}$ and its beacon position $\left(x_{i}, y_{i}\right)$ is close to $\left(x_{0}, y_{0}\right)$.

- As we will discuss later in Theorem 1, more than half of all intersection points are concentrated inside a certain circle, called boundary circle. The center of the boundary circle is $\left(x_{0}, y_{0}\right)$ and its area is relatively small compared to the entire area. Intuitively, the reason is that the distances between the circle lines of the benign references and $\left(x_{0}, y_{0}\right)$ are smaller than $\epsilon$.

Based on these two facts, we can see that the more harmful an inside-attack reference is, the more intersection points are included by the reference. For example, in Fig. 3, the numbers of intersection points included by the benign references $l_{1}, l_{2}, l_{3}$ and $l_{4}$ are $3,5,5$ and 3 , respectively. However, the inside-attack references $l_{5}$ and $l_{6}$ include 15 and 14 intersection points respectively. The reason is that the inside-attack references include almost all intersection points of the benign references. However, the benign references may not include intersection points made by inside-attack references.

In addition, even if the fraction of inside-attack references increases, this observation still is valid. Let $p_{i}^{b n}$ be the number of intersection points included by a benign reference and $p_{j}^{i n}$ be the number of intersection points included by an inside-attack reference. If the fraction of inside-attack references increases, both $p_{i}^{b n}$ and $p_{j}^{i n}$ decrease with a high probability. The example in Fig. 4 is the same as the example of Fig. 3 except for $l_{6}$ which is benign in the example in Fig. 4, but is malicious in the example in Fig. 3. In Fig. 4, $\left\{p_{1}^{b n}, p_{2}^{b n}, p_{3}^{b n}, p_{4}^{b n}, p_{5}^{i n}, p_{6}^{b n}\right\}=\{5,7,7,5,18,9\}$, whereas in Fig. 3, $\left\{p_{1}^{b n}, p_{2}^{b n}, p_{3}^{b n}, p_{4}^{b n}, p_{5}^{i n}, p_{6}^{i n}\right\}=\{3,5,5,3,15,14\}$. After $l_{6}$ becomes malicious, both $p_{i}^{b n}$ and $p_{j}^{i n}$ for all $i$ and $j$ decrease except $p_{6}^{i n}$. The reason is that the intersection points made by $l_{6}$ with benign references are removed.

DeFinition 2. (Outer reference) Given a location reference $\left(x_{i}, y_{i}, d_{i}\right)$, its outer reference is $\left(x_{i}, y_{i}, d_{i}+\epsilon\right)$, where $\epsilon$ is the maximum measurement error. The benign outer references must include the real sensor position.

Definition 3. (Boundary circle) The boundary circle is a circle with center equal to the true sensor position and radius equal to $\sqrt{d_{\max }^{2}-\left(d_{\max }-\epsilon\right)^{2}}=\sqrt{2 \epsilon d_{\max }-\epsilon^{2}}$, where $d_{\text {max }}$ is the maximum measurement distance.

LEMMA 1. Inside the boundary circle, there is at least one of two intersection points made by two outer benign references.

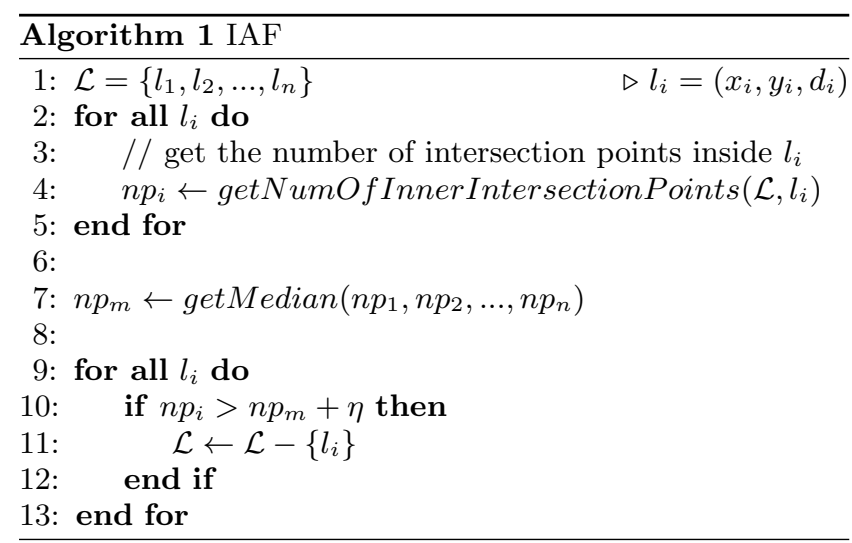

PROOF. We refer the reader to [13].

THEOREM 1. The number of intersection points inside the boundary circle is at least $\left(\begin{array}{l}n \\ 2\end{array}\right)(=n \times(n-1) / 2)$.

Proof. The number of all intersection points is $\left(\begin{array}{l}n \\ 2\end{array}\right) \times$ 2. Since at least one of them is inside the boundary circle according to Lemma 1 , the number of intersection points inside the boundary circle is at least $\left(\begin{array}{l}n \\ 2\end{array}\right) \times 2 \times 1 / 2=\left(\begin{array}{c}n \\ 2\end{array}\right)$.

Based on the above observations, we developed a heuristic filtering algorithm called Inside-Attack Filtering (IAF). The algorithm works as follows:

1. It obtains the intersection points of all location references.

2. For each location reference $l_{i}$, it counts the number $n p_{i}$ of intersection points included in $l_{i}$.

3. It computes the median $n p_{m}$ of $\left\{n p_{1}, n p_{2} \ldots, n p_{n}\right\}$.

4. It removes the location references for which $n p_{i}$ is larger than $n p_{m}$ plus a threshold $\eta$.

The pseudo-code of IAF is shown in Algorithm 1. In step 3 (code-line 7 ), we utilize the median instead of the mean since the median provides a more representative value when there are outliers. $\eta$ is a system parameter used to adjust the filtering sensitivity. If $\eta$ decreases, the detection rate increases, but the false positive rate also increases. On the other hand, if $\eta$ increases, the false positive rate decreases, but the detection rate also decreases. $\eta$ is set as follows:

$$
\eta=\left(\begin{array}{c}
n^{\prime} \\
2
\end{array}\right)-\left(\begin{array}{c}
n^{\prime}-1 \\
2
\end{array}\right)+\alpha,
$$

where $n^{\prime}=\lceil n / 2\rceil$ and $\alpha \geq 0 . n$ is the number of location references. For instance, if $n$ is 7 and $\alpha=0, \eta=\left(\begin{array}{l}4 \\ 2\end{array}\right)-\left(\begin{array}{l}3 \\ 2\end{array}\right)=3$. When $n$ is 7 , there are at least 4 benign references and there are at least $\left(\begin{array}{l}4 \\ 2\end{array}\right)$ intersection points inside the boundary circle, which means that the inside-attack references include at least $\left(\begin{array}{l}4 \\ 2\end{array}\right)$ intersection points if they are bigger than the boundary circle (Theorem 1). On the other hand, a benign reference approximately includes $\left(\begin{array}{l}3 \\ 2\end{array}\right)$ intersection points which are made by 3 other benign references.

$\alpha(\geq 0)$ is added in order to reduce the false positive rate, i.e., to prevent benign references from being removed by IAF. $\eta$ without $\alpha$ is very conservative since it assumes the worst case, i.e., that $\lfloor n / 2\rfloor$ of location references are malicious. If $\alpha$ is small, IAF will aggressively filter out all 


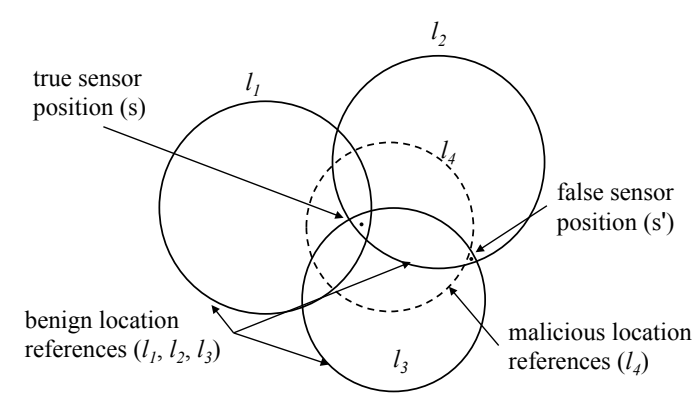

Figure 5: An example showing that IAF-MMSE cannot filter out a malicious location reference.

malicious references, but sometimes it will remove benign ones (high true/false positive rate). On the other hand, if $\alpha$ is large, IAF will loosely filter out malicious references (low true/false positive rate). Notice that even if some malicious references are not filtered out by IAF, the unfiltered malicious ones can be filtered out by the rest of our entire IAF-MMSE algorithm discussed in Sec. 5.2.

\subsection{IAF-MMSE}

To estimate sensor positions, we propose the IAF-MMSE algorithm. Like EAR-MMSE, IAF-MMSE checks the DOCs of location references. However, IAF-MMSE can remove multiple malicious references in one round, whereas EARMMSE removes one reference per round. Therefore, IAFMMSE is able to reduce the number of MMSE operations. IAF-MMSE consists of three steps.

1. As shown in Algorithm 2, IAF-MMSE first removes all obviously malicious references based on the assumption that the majority of references is benign. In other words, if there are $n$ references and more than half of them are benign, the DOC of a benign reference must be greater than or equal to $\lfloor n / 2\rfloor$. For example, if $n$ is 7 and four of them are benign, the DOCs of the benign references must be at least 3 . If a reference has a DOC of 2 , it is removed since it is obviously malicious (Line $1 \sim 7$ ).

2. Inside-attack references are removed by IAF (Line 9).

3. After the inside-attack references are removed, IAF-MMSE iteratively runs MMSE using the remaining references similarly to EAR-MMSE. However, if multiple references are obviously malicious, they are removed simultaneously. This procedure is repeated until $\delta$ is smaller than $\theta$ or the number of remaining location references is less than 3. (Line 11 30). The simultaneous removal reduces the number of MMSE executions and thus increases the computational efficiency.

\subsection{Security Analysis}

IAF-MMSE can estimate a sensor position by removing the effect of the malicious location references when there are more benign references than malicious ones. To defeat IAFMMSE, the attacker must first generate inside-attack references satisfying the conditions introduced in Sec. 4.1. If an inside-attack reference does not satisfy the conditions, it will be filtered out due to the lack of DOC. Then, the attacker must decrease the measurement distance of an inside-attack

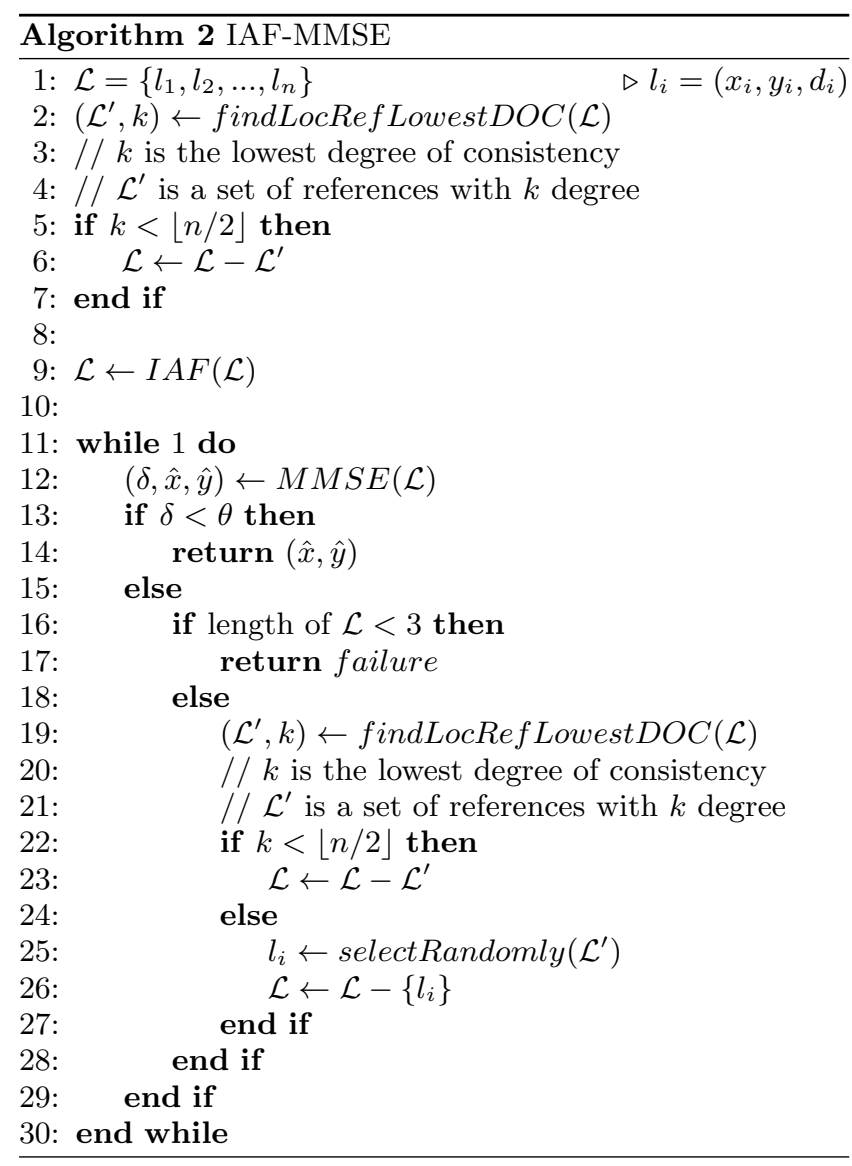

reference $l_{i}$ to reduce the number of intersection points inside $l_{i}$. In this case, although the probability that IAF filters out $l_{i}$ decreases, the maliciousness of $l_{i}$ also decreases at the same time.

IAF-MMSE obviously has a limit when all references have the same DOC and have a similar number of intersection points. As shown in Fig. 5, if a malicious location reference $\left(l_{4}\right)$ is accidentally consistent with benign references $\left(l_{2}\right.$ and $\left.l_{3}\right)$, the estimated location will be $s^{\prime}$ (false sensor location) rather than $s$ (true sensor location) since DOCs of $l_{1}, l_{2}, l_{3}$ and $l_{4}$ are equal to 3 . In addition, $l_{1}, l_{2}, l_{3}$ and $l_{4}$ include same number of intersection points. However, in such cases, any state-of-the-art secure localization techniques $[10$, 12] cannot correctly estimate the true sensor position since $\left\{l_{2}, l_{3}, l_{4}\right\}$ looks more consistent than $\left\{l_{1}, l_{2}, l_{3}\right\}$.

\section{EVALUATION}

In this section, we evaluate IAF-MMSE through simulations, and compare it with other MMSE-based algorithms: BF-MMSE [10], GAR-MMSE [10], EAR-MMSE [10], and CMMSE [12]. We developed a simulator specialized for sensor localization. Since the considered algorithms are purely based on location references, we did not use general network simulators such as NS-2 [2]. For all simulations, a target sensor $\left(x_{0}, y_{0}\right)$ is located at the center of a $30 \mathrm{~m} \times 30 \mathrm{~m}$ area. 4 benign beacon nodes are randomly deployed in the area. The number of malicious (inside-attack) references is set to 3 . The beacon position $\left(x_{i}, y_{i}\right)$ of an inside-attack reference $l_{i}$ is randomly located within $1.5 \mathrm{~m}$ away from true sensor po- 


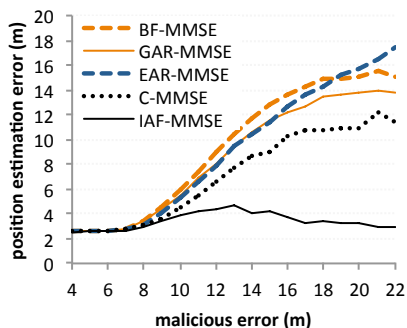

(a) Sensor position estimation error

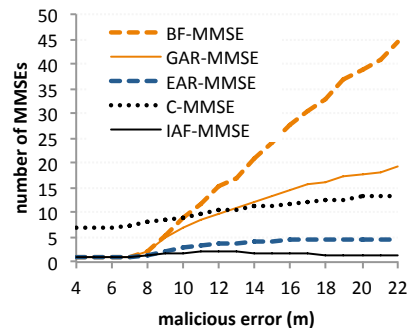

(b) Number of MMSE opera-

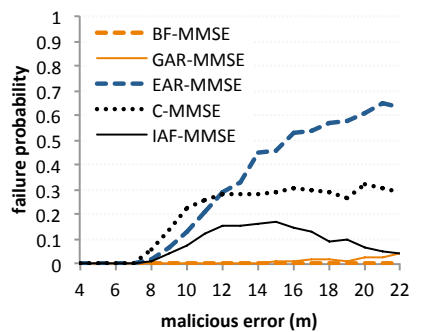

(c) Failure probability

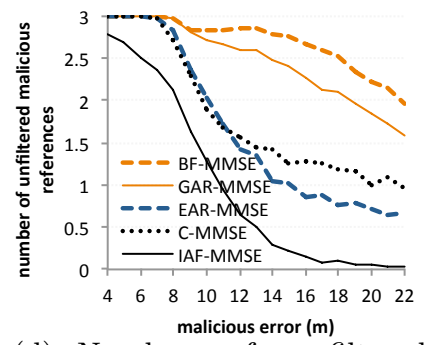

(d) Number of unfiltered inside-attack references

Figure 6: Simulation results (three inside-attack references exist).

sition. In this scenario, malicious error refers to a malicious modification of the measured distance $d_{i}$ in an inside-attack reference. The maximum distance measurement $d_{\max }$ is set to $26 \mathrm{~m}$. The distance measurement errors are uniformly distributed in $[-\epsilon, \epsilon]$ like in $[10,16,15]$ and $\epsilon$ is set to $4 m$. The threshold $\eta$ for IAF is set to 5 . We utilize the basic MMSE [11] and the threshold $\theta$ is set to $\epsilon^{2}$.

\subsection{Simulation Results}

As shown in Fig. 6(a), IAF-MMSE is the most accurate algorithm and its accuracy does not decrease much even when the malicious error increases. Interestingly, the accuracy of BF-MMSE and GAR-MMSE as well as EAR-MMSE severely degrades as the malicious error increases. However, the reasons for the accuracy degradation are different. EAR-MMSE, as shown in Fig. 6(c), has the highest failure rate since it cannot distinguish malicious references from benign references and thus removes benign references with high probability. In the case of BF-MMSE and GAR-MMSE, although their failure rates are low, the unfiltered rates (see Fig. 6(d)) are higher than other schemes, which means that BF-MMSE and GAR-MMSE seldom filter out inside-attack references. They just try to estimate the best sensor location with all references, which results in large estimation errors.

In terms of the number of MMSE operations, IAF-MMSE shows the best performance (see Fig. 6(b)). When there are 3 inside-attack references and the malicious error is $22 m$, IAF-MMSE requires only 1.2 MMSE operations, whereas EAR-MMSE requires 4.7 MMSE operations on average. The reason is that IAF-MMSE removes inside-attack references before MMSE is iteratively executed.

\section{CONCLUSION}

In this paper, we introduced the inside attack by which an attacker can severely distort the estimated locations of sensors if the attacker knows the locations of the sensors. We found that most state-of-the-art MMSE-based schemes are vulnerable to such attacks. To defend against this attack, we proposed the inside-attack filtering algorithm which filters out malicious location references based on the number of intersection points. We also propose an efficient algorithm which estimates sensor positions when the number of benign location references is larger than the number of malicious location references.

\section{Acknowledgments}

The work reported in this paper has been partially supported by the Purdue Cyber Center and by the National Science Foundation under grant CNS-1111512.

\section{REFERENCES}

[1] Atmel, http://www.atmel.com/images/doc8228.pdf.

[2] ns2, http://www.isi.edu/nsnam/ns/.

[3] S. Capkun and J.-P. Hubaux. Secure positioning in wireless networks. Selected Areas in Communications, IEEE Journal on, 24(2):221-232, Feb 2006.

[4] B. Cheng, R. Hudson, F. Lorenzelli, L. Vandenberghe, and K. Yao. Distributed gauss-newton method for node loclaization in wireless sensor networks. In IEEE SPAWC, 2005.

[5] R. Garg, A. Varna, and M. Wu. Gradient descent approach for secure localization in resource constrained wireless sensor networks. In IEEE Acoustics Speech and Signal Processing, 2010.

[6] B. Karp and H. T. Kung. Gpsr: Greedy perimeter stateless routing for wireless networks. In $A C M$ MobiCom, 2000.

[7] L. Lazos, R. Poovendran, and S. Capkun. Rope: robust position estimation in wireless sensor networks. In IPSN, 2005.

[8] Z. Li, W. Trappe, Y. Zhang, and B. Nath. Robust statistical methods for securing wireless localization in sensor networks. In IPSN, 2005.

[9] D. Liu and P. Ning. Location-based pairwise key establishments for static sensor networks. In $A C M$ SASN, 2003.

[10] D. Liu, P. Ning, A. Liu, C. Wang, and W. K. Du. Attack-resistant location estimation in wireless sensor networks. ACM TISSEC, 11(4):22:1-22:39, July 2008.

[11] A. Savvides, C.-C. Han, and M. B. Strivastava. Dynamic fine-grained localization in ad-hoc networks of sensors. In Proceedings of MobiCom, 2001.

[12] C. Wang, A. Liu, and P. Ning. Cluster-based minimum mean square estimation for secure and resilient localization in wireless sensor networks. In WASA, 2007.

[13] J. Won and E. Bertino. The full version of this paper. available from the authors.

[14] J. Won and E. Bertino. Inside attack filtering for robust sensor localization. In IEEE ICDCS, pages 796-797, June 2015.

[15] Y. Zeng, J. Cao, S. Zhang, S. Guo, and L. Xie. Pollution attack: A new attack against localization in wireless sensor networks. In IEEE WCNC, 2009.

[16] S. Zhong, M. Jadliwala, S. Upadhyaya, and C. Qiao. Towards a theory of robust localization against malicious beacon nodes. In IEEE INFOCOM, 2008. 